\title{
Seronegative Neuromyelitis Optica Spectrum Disorder following Exposure to Hepatitis B Vaccination
}

\author{
Richard Heekin Chetan Gandhy Derrick Robertson \\ Department of Neurology, University of South Florida Morsani College of Medicine, \\ Tampa, Fla., USA
}

\section{Key Words}

Neuromyelitis optica · Hepatitis B vaccine - Demyelination · Optic neuritis · Transverse myelitis

\begin{abstract}
Controversy exists regarding a potential link between exposure to recombinant hepatitis $B$ vaccine (HBV) and central nervous system demyelinating diseases. Here, we present a case of seronegative neuromyelitis optica spectrum disorder (NMOSD) following exposure to HBV. A 28-year-old man developed painful eye movements 11 days after exposure to HBV. Within $24 \mathrm{~h}$, he experienced vision loss, ascending numbness, and ataxia. T-spine MRI showed a cord lesion spanning T6-T9. Brain MRI showed bilateral optic nerve contrast enhancement and a right-sided internal capsule lesion. Cerebrospinal fluid analysis was normal, including negative oligoclonal bands and normal IgG index. AQP4-IgG serology was negative. The patient's visual symptoms improved after treatment with steroids and plasma exchange. He received plasma exchange weekly for 4 weeks with decreased numbness and tingling as well as improved coordination. Treatment with mycophenolate mofetil was started, and the patient remains clinically stable with near resolution of his prior symptoms. Neuromyelitis opti$\mathrm{ca}$ is characterized by optic neuritis and/or longitudinally extensive transverse myelitis. While our patient tested seronegative for AQP4-IgG (which remains negative in 10-50\% of NMOSD cases, despite testing with the most sensitive assays available), he did meet NMOSD diagnostic criteria. In a literature review, we found 7 cases of NMOSD onset or relapse associated with exposure to various vaccines, but to our knowledge this represents the first published report of NMOSD onset following exposure to HBV. While causality between vaccination and CNS demyelinating disease remains elusive, it is important to report these cases to help develop safer vaccinations and provoke further inquiry into the pathogenesis of NMOSD.
\end{abstract}

(C) 2015 S. Karger AG, Basel 
Heekin et al.: Seronegative Neuromyelitis Optica Spectrum Disorder following Exposure to Hepatitis B Vaccination

\section{Introduction}

Since the mid-1990s, controversy has surrounded the question of a potential link between exposure to recombinant hepatitis B vaccine (HBV) and demyelinating diseases of the central nervous system (CNS), in particular multiple sclerosis (MS). While most epidemiological studies have failed to demonstrate a link between HBV and CNS demyelinating disease, a recent systematic review concluded that, in light of the methodological limitations of these studies, it is not possible at present to determine whether exposure to the vaccine is associated with an increased risk of MS onset or relapse, or of other CNS demyelinating diseases [1]. Numerous case reports exist that describe a temporal relationship between HBV administration and the onset of CNS demyelinating diseases, including MS, acute disseminated encephalomyelitis, optic neuritis both unilateral and bilateral, and transverse myelitis [1-5]. Here, we present the case of a patient who developed seronegative neuromyelitis optica spectrum disorder (NMOSD) following exposure to HBV.

\section{Case Presentation}

The patient, a 28-year-old male graduate student with no relevant past medical history, developed pain with eye movements bilaterally 11 days after receiving HBV and Tdap (tetanus-diphtheria-acellular pertussis) vaccines before the start of the academic semester. Within $24 \mathrm{~h}$ of eye pain onset, he experienced decreased vision in both eyes, progressing to complete loss of vision within 1 day. This was accompanied by ascending numbness and tingling from his feet to his mid-thoracic region and associated with fatigue, incoordination, and loss of balance; however, his strength remained intact. He was admitted to the hospital where MRI of the thoracic spine showed a large nonenhancing lesion involving the right side of the spinal cord extending from $\mathrm{T} 6$ through $\mathrm{T} 9$, as well as a second smaller lesion involving the posterior central thoracic spinal cord at the level of T5 (fig. 1). MRI of the brain showed contrast enhancement of bilateral optic nerves on T1-weighted imaging consistent with optic neuritis (fig. 2) and a nonenhancing T2 lesion with restricted diffusion at the junction of the right thalamus and posterior limb of the internal capsule (fig. 3). Cerebrospinal fluid analysis revealed a cell count with differential, $1 / \mu \mathrm{l}$, that was within normal limits, absence of oligoclonal bands, and normal glucose, protein, and IgG index. Aquaporin-4 (AQP4) IgG autoantibodies in the serum were negative by enzyme-linked immunosorbent assay (ELISA). The patient received both intravenous steroids and 5 sessions of plasma exchange. He remained in the hospital for 1 week, and prior to discharge, his visual symptoms began to improve. Following discharge, the patient received plasma exchange weekly for 4 additional treatments. At the time he presented to our clinic, which was approximately 3 weeks after discharge from the hospital, his vision had continued to improve, with restoration of color vision and shapes/details, although the vision in his left eye remained subjectively 'darker' compared to the right eye. At that time, his numbness and tingling had also significantly decreased, with improved balance and coordination. The diagnosis of optic neuritis was based on clinical examination findings of bilateral relative afferent pupillary defects, bilateral optic disc pallor, and the MRI finding of enhancement of the optic nerves. The patient was started on mycophenolate mofetil. The patient has relocated out of state for further college education. He now follows with a colleague who informed us that as of 9 months after clinical presentation, the patient remains free of new neurological symptoms, with continued improvement of visual acuity and near-complete resolution of numbness, tingling and balance difficulties. He continues on mycophenolate mofetil, which he has tolerated well. The patient 
Heekin et al.: Seronegative Neuromyelitis Optica Spectrum Disorder following Exposure to Hepatitis B Vaccination

had a follow-up MRI of the entire neuraxis 9 months after initial presentation that demonstrated no new lesions and stability of prior lesions.

\section{Discussion}

Neuromyelitis optica (NMO) is characterized by severe optic neuritis and/or longitudinally extensive transverse myelitis, defined as a T2 lesion extending over $>3$ vertebral segments, in association with brain MRI findings at onset not fulfilling the diagnostic criteria for MS. The discovery of AQP4-IgG autoantibodies, a sensitive and highly specific biomarker for NMO which likely plays a role in pathogenesis, has led to the recognition of a wider spectrum of the disease, now referred to as NMOSD [6]. While the detection of AQP4-IgG autoantibodies can confirm a diagnosis of NMOSD, about $10-50 \%$ of patients with NMOSD remain negative for these antibodies in spite of testing with the most sensitive assays currently available $[6,7]$. Our patient tested negative for AQP4-IgG with the commercially available ELISA; however, it should be noted that other tests exist for AQP4-IgG autoantibodies with greater sensitivity and specificity. In the study by Sato et al. [8], approximately $20 \%$ of patients positive for AQP4-IgG autoantibodies by cell-based assay using M23 isoform transfected living HEK293 cells were negative with ELISA. In another study, the Mayo group compared various commercial and in-house assays, finding that $61 \%$ of NMO cases previously classified as seronegative tested positive for AQP4-IgG autoantibodies with the more sensitive assays [9]. While MS cannot be excluded entirely in our patient due to the lack of AQP4-IgG antibodies, his clinical presentation better satisfies the NMOSD criteria given the presence of bilateral optic neuritis, a longitudinally extensive lesion spanning three segments in his thoracic spine, and an MRI of the brain that is nondiagnostic for MS. In addition, there is the possibility that this a case of anti-myelin oligodendrocyte glycoprotein (anti-MOG)-associated NMOSD. We were unable to test for this antibody as the patient relocated. Furthermore, testing for anti-MOG antibodies is currently considered experimental and investigational and therefore not readily available outside of research settings [10].

While previous case reports have described temporal associations between several CNS demyelinating diseases and preceding exposure to recombinant HBV, to the best of our knowledge this case represents the first published report of NMOSD onset following administration of HBV. A recent review of the literature from 1979 to 2013 found 7 cases of NMOSD onset or relapse (both seropositive and seronegative for AQP4-IgG) associated with exposure to various vaccines, with the vaccine against human papilloma virus being the most commonly implicated [11]. One potential limitation of this case is that our patient also received the Tdap vaccine prior to NMOSD onset; however, we think this is unlikely to be of pathogenic significance given that we were unable to find any published reports linking Tdap exposure to CNS demyelinating disease. Although it is difficult, if not impossible, to establish a causal relationship between vaccination and CNS demyelinating disease through the temporal association of rare events described in case reports such as this, we nevertheless believe it is important to make such cases known, both to contribute to the continued development of safe and efficacious methods of vaccination, as well as to provoke further investigation into the pathogenesis of NMOSD, which may lead to a more refined nosology of this evolving spectrum of disease. 
Heekin et al.: Seronegative Neuromyelitis Optica Spectrum Disorder following Exposure to Hepatitis B Vaccination

\section{Disclosure Statement}

R.H. has no potential conflicts of interest to report. C.G. has served as a consultant for Teva Neuroscience. D.R. has served as a consultant for Biogen Idec, Genzyme/Sanofi Aventis, Teva Neuroscience and Pfizer; is on the speakers' bureau for Biogen Idec, Pfizer, EMD Serono, Genzyme/Sanofi Aventis, Novartis, Teva Neuroscience, Mallinckrodt and Acorda, and has received grant support from Biogen Idec, Genzyme/Sanofi Aventis, Novartis, Sun Pharma, MedImmune, GlaxoSmithKline, and Roche/Genetech.

\section{References}

-1 Martínez-Sernández V, Figueiras A: Central nervous system demyelinating diseases and recombinant hepatitis B vaccination: a critical systematic review of scientific production. J Neurol 2013;260:1951-1959.

-2 Brinar VV, Poser CM: Disseminated encephalomyelitis in adults. Clin Neurol Neurosurg 2008;110:913-918.

-3 Stewart 0, Chang B, Bradbury J: Simultaneous administration of hepatitis B and polio vaccines associated with bilateral optic neuritis. Br J Ophthalmol 1999;83:1200-1201.

-4 Tartaglino LM, Heiman-patterson T, Friedman DP, Flanders AE: MR imaging in a case of postvaccination myelitis. AJNR Am J Neuroradiol 1995;16:581-582.

5 Trevisani F, Gattinara GC, Caraceni P, et al: Transverse myelitis following hepatitis B vaccination. J Hepatol 1993;19:317-318.

-6 Flanagan EP, Weinshenker BG: Neuromyelitis optica spectrum disorders. Curr Neurol Neurosci Rep 2014;14:483.

-7 Sato DK, Callegaro D, Lana-peixoto MA, Nakashima I, Fujihara K: Seronegative neuromyelitis optica spectrum - the challenges on disease definition and pathogenesis. Arq Neuropsiquiatr 2014;72:445-450.

-8 Sato DK, Nakashima I, Takahashi T, et al: Aquaporin-4 antibody-positive cases beyond current diagnostic criteria for NMO spectrum disorders. Neurology 2013;80:2210-2216.

$\$ 9$ Jiao Y, Fryer JP, Lennon VA, et al: Updated estimate of AQP4-IgG serostatus and disability outcome in neuromyelitis optica. Neurology 2013;81:1197-1204.

-10 Mao Z, Lu Z, Hu X: Distinction between MOG antibody-positive and AQP4 antibody-positive NMO spectrum disorders. Neurology 2014;83:1122.

-11 Karussis D, Petrou P: The spectrum of post-vaccination inflammatory CNS demyelinating syndromes. Autoimmun Rev 2014;13:215-224. 


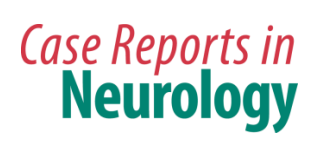

\begin{tabular}{l|l}
\hline \multicolumn{2}{l|}{ Case Rep Neurol 2015;7:78-83 } \\
\hline DOI: $10.1159 / 000381826$ & $\begin{array}{l}\text { C } 2015 \text { S. Karger AG, Basel } \\
\text { www.karger.com/crn }\end{array}$ \\
\hline
\end{tabular}

Heekin et al.: Seronegative Neuromyelitis Optica Spectrum Disorder following Exposure to Hepatitis B Vaccination

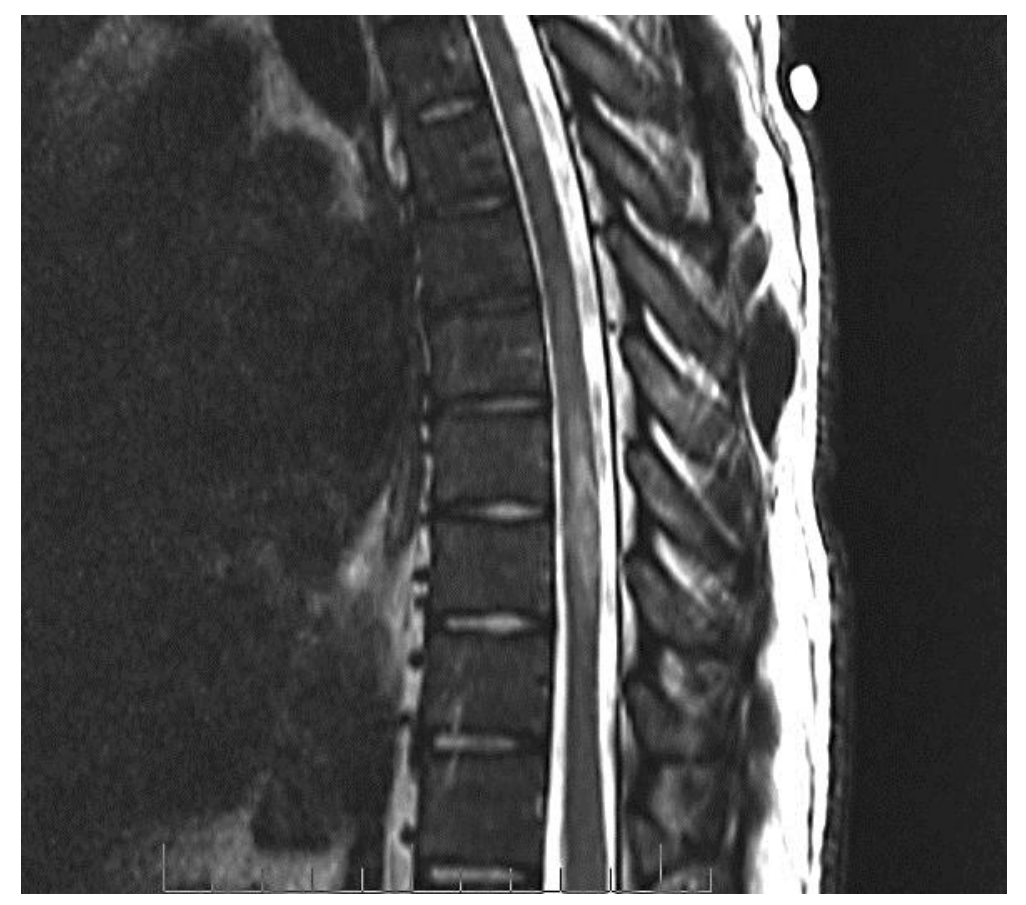

Fig. 1. MRI of the thoracic spine: sagittal T2 sequence partially demonstrating a hyperintenseT6-T9 intramedullary lesion along with an isolated hyperintense T5 lesion.

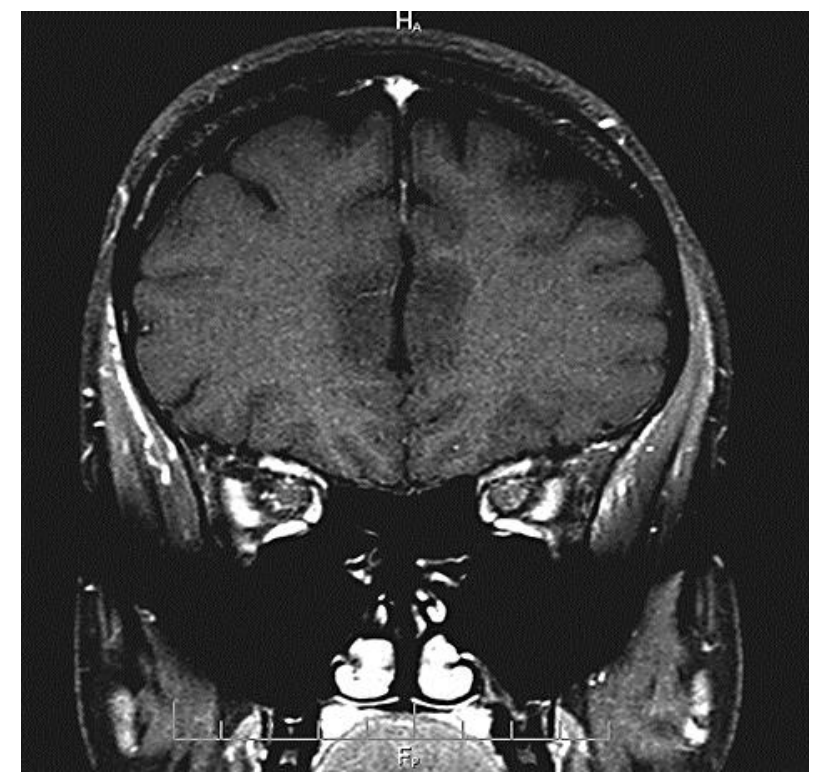

Fig. 2. MRI of the brain: T1 coronal post-contrast sequence demonstrating faint bilateral optic nerve enhancement. 


\section{Case Reports in Neurology}

\begin{tabular}{l|l}
\hline Case Rep Neurol 2015;7:78-83 \\
\hline DOI: $10.1159 / 000381826$ & $\begin{array}{l}\text { C 2015 S. Karger AG, Basel } \\
\text { www.karger.com/crn }\end{array}$ \\
\hline
\end{tabular}

Heekin et al.: Seronegative Neuromyelitis Optica Spectrum Disorder following Exposure to Hepatitis B Vaccination

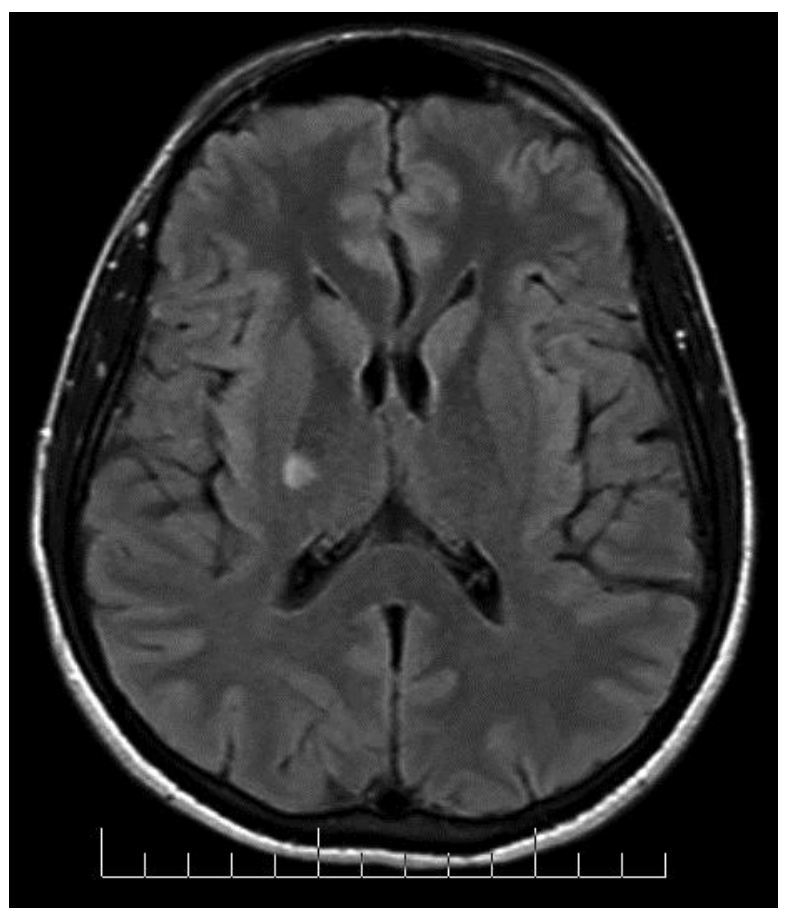

Fig. 3. MRI of the brain: T2 FLAIR axial sequence demonstrating a solitary hyperintense lesion at the junction of the right thalamus and the posterior limb of the internal capsule. 